\title{
Clinical Significance of Histologic Variants of Bladder Cancer
}

\author{
Joshua I. Warrick, MD
}

\begin{abstract}
Pathologists have identified many "histologic variants" of bladder cancer (BCA): histologic patterns that differ from conventional urothelial carcinoma (transitional cell carcinoma). Several of these are biologically aggressive, and their identification may aid in clinical decisionmaking. This article reviews several histologic variants and their value in deciding management of cT1 disease and predicting response to neoadjuvant chemotherapy (NAC). Diagnostic issues are also addressed, such as interobserver variability among pathologists. For example, although stage CT1 conventional urothelial carcinoma is usually managed conservatively, cT1 micropapillary carcinoma has high mortality following conservative management, and early cystectomy may reduce mortality. Similarly, plasmacytoid and small cell cancers are remarkably aggressive, and those diagnosed as stage CT1 at transurethral resection are likely understaged; conservative management thus greatly risks undertreatment. As an example of response, NAC dramatically reduces mortality in patients with small cell BCA, and is thus the standard of care, even in stage CT1 disease. Although identification of histologic variants may inform on optimal management, diagnostic issues challenge their incorporation into clinical practice. For example, interobserver reproducibility is only moderate for the diagnosis of micropapillary BCA. Studies have identified specific histologic issues underlying this diagnostic irreproducibility, and ongoing work aims to remedy this issue. In summary, histologic variants are emerging as potentially useful biomarkers in the management of BCA. Although issues remain unresolved, pathologists and treating physicians will benefit from understanding these variants and their prognostic and therapeutic implications.
\end{abstract}

J Natl Compr Canc Netw 2017;15(10):1268-1274 doi: 10.6004/jnccn.2017.7027

Pathologists have identified many bladder cancer (BCA) histomorphologies that differ from conventional urothelial carcinoma (UC; also known as transitional cell carcinoma). Several of these histologic variants are biologically aggressive, and their identification may aid in clinical decision-making.

Tumor stage drives BCA management. Treatment is typically conservative in patients with non-muscleinvasive (NMI) tumors diagnosed on biopsy or transurethral resection, including noninvasive cancers (stage cTa and cTis) and invasive cancers limited to the lamina propria (stage cT1). ${ }^{1}$ Conservative treatment entails transurethral resection of all cystoscopically visible tumor, often followed by intravesical therapy, such as bacillus Calmette-Guérin (BCG); morbidity is minimal, bladder function is preserved, and overall mortality is low. However, although mortality is very low $(<5 \%)$ in

From the Departments of Pathology and Surgery, Division of Urology, Penn State Health Milton S. Hershey Medical Center, Hershey, Pennsylvania. Submitted April 26, 2017; accepted for publication August 23, 2017.

The author has disclosed that he has no financial interests, arrangements, patients with low-risk NMI BCA, ${ }^{2}$ mortality is considerably higher in those with high-risk NMI tumors, a group that includes all stage cT1 cases. Approximately $30 \%$ of conservatively managed patients with high-risk cancers will die of the disease on long-term follow-up (10-15 years), and only a quarter will maintain an intact, functional bladder in the long term. ${ }^{3}$ Definitive treatment of patients with cT1 disease is radical cystectomy (early cystectomy), which improves mortality compared with conservative management. ${ }^{3}$ However, cystectomy entails considerable functional morbidity. Treatment of cT1 disease is thus a difficult balance between preventing mortality and preserving the patient's normal urinary tract function.

BCA that invades the muscularis propria ( $\geq$ stage cT2) kills most patients if managed conservatively. ${ }^{4}$ Patients with such muscle-invasive cancers are thus 
Histologic Variants of Bladder Cancer

managed definitively, typically with radical cystectomy, which improves survival. ${ }^{4}$ Survival is further improved by the addition of neoadjuvant chemotherapy (NAC), usually gemcitabine and cisplatin, or MVAC (methotrexate/vinblastine/doxorubicin/ cisplatin). ${ }^{5}$ However, the survival benefit is largely limited to patients in whom the tumor histologically responds to chemotherapy, typically defined as a complete pathologic response (cPR) - the complete absence of cancer on histologic examination of the cystectomy specimen. ${ }^{5}$ Only $30 \%$ to $40 \%$ of patients experience $\mathrm{cPR}$, and 3.7 patients must receive NAC to save 1 life. ${ }^{5} \mathrm{NAC}$ also delays surgery by several months and has its own risks, including potential nephrotoxicity and neurotoxicity. Many patients thus endure a delay in definitive management that is nonbeneficial and potentially harmful.

This article serves 2 purposes. First, it reviews the therapeutic and prognostic significance of histologic variants of BCA, with specific emphasis on the decisions to perform early cystectomy in cT1 disease and to give NAC in muscle-invasive disease (Table 1). Second, it reviews diagnostic issues with histologic variants, such as interobserver variability among pathologists. Seven histologic variants are reviewed: micropapillary, plasmacytoid, small cell, squamous, glandular, nested, and lymphoepithelioma-like (Figure 1). A few general observations apply to these variants and will constrain the discussion. First, with the exception of squamous morphology, these histologic variants are uncommon, each accounting for $<10 \%$ of BCA diagnoses. ${ }^{6}$ Studies with large cohorts are thus difficult to assemble, and most evidence comes from smaller retrospective studies.
Second, these variants often co-occur with conventional UC. Tumors with pure squamous and pure glandular morphology have been designated "pure squamous cell carcinoma" and "pure adenocarcinoma," respectively, for many years, ${ }^{7}$ and this review adheres to this convention. Studies of lymphoepithelioma-like cancer have also consistently distinguished between cancers with focal variants and those with pure or predominant variants, and this review recognizes this distinction. However, for the remaining variants, several studies have shown that mixed cases (histologic variant with conventional UC) have similar clinical behavior to pure histologic variants. ${ }^{8-11}$ This is somewhat of an oversimplification. For example, increasing fraction of micropapillary morphology in a given case is associated with worse outcome. ${ }^{12}$ However, for the sake of uniformity, this article does not distinguish mixed from pure variants for micropapillary, plasmacytoid, small cell, or nested carcinomas. Third, specific variants have specific clinical and management associations. Many prior studies considered all histologic variants as a single category. This review focuses on studies that separate specific variants. Fourth, histomorphologic descriptions in this review are somewhat abbreviated, because the aim is to discuss the clinical significance of histologic variants. Excellent reviews and book chapters have been written on the histomorphologic details of these variants, ${ }^{13-15}$ and this article will not reproduce their work. Lastly, this review is limited to invasive cancers arising from the bladder proper; noninvasive neoplasia and urachal neoplasia will not be covered.

\section{Table 1. Optimal Treatment of Histologic Variants of Bladder Cancer}

\begin{tabular}{|lll|} 
& Early Cystectomy in cT1 Cancer & $\begin{array}{l}\text { Neoadjuvant Chemotherapy Benefit in } \\
\text { Muscle-Invasive Disease }\end{array}$ \\
\hline Micropapillary & Controversial & Probable $^{\text {b }}$ \\
\hline Plasmacytoid & Yes & Unknown \\
\hline Small cell & Yes & Yes \\
\hline Pure squamous cell carcinoma & Yes & Unknown \\
\hline UC with squamous differentiation & No & Yes \\
\hline Pure adenocarcinoma & Yes & Unknown \\
\hline UC with glandular differentiation & No & Yes \\
\hline Nested & Unknown & Unknown \\
\hline
\end{tabular}

Abbreviation: UC, urothelial carcinoma.

a Opinions differ on the appropriate management of CT1 micropapillary bladder cancer, particularly given diagnostic reproducibility issues (see

"Micropapillary" discussion, page 1270).

'Evidence shows micropapillary cancer histologically responds to neoadjuvant chemotherapy, suggesting improved survival, though survival benefit has not directly been shown. 

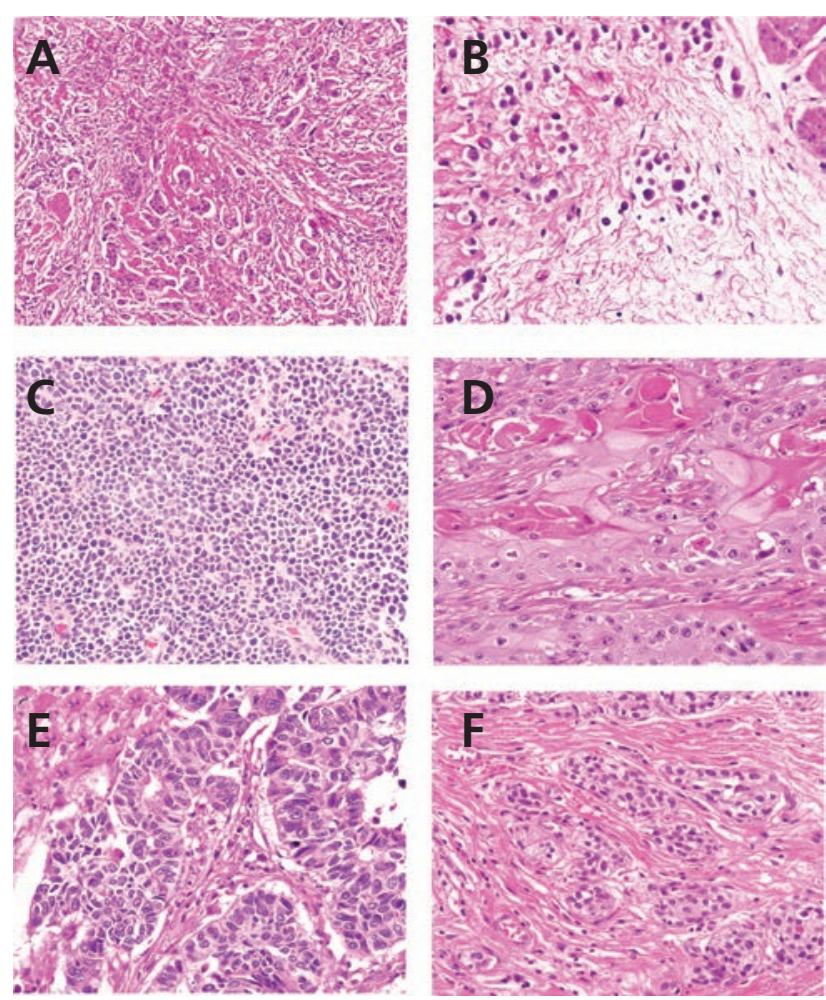

Figure 1. Hematoxylin-eosin-stained sections of histologic variants. (A) Micropapillary morphology (original magnification, x200) appears as small invasive nests with surrounding tissue retraction. (B) Plasmacytoid cancer (x400) seen as infiltrative single cells. (C) Small cell cancer (x400) has high nuclear-to-cytoplasmic ratio, nuclear molding, and numerous mitotic figures. (D) Squamous carcinoma $(x 400)$ seen as keratin formation within invasive carcinoma. (E) Glandular morphology (x400) appears as formation of true glandular structures within invasive cancer. (F) Nested cancer $(x 400)$ consists of infiltrative small nests of tumor cells with minimal cytologic atypia.

\section{Histologic Variants}

\section{Micropapillary}

Micropapillary morphology describes invasive small cancer nests with surrounding tissue retraction. ${ }^{7,12}$ This variant is clinically aggressive, typically presenting with high tumor stage and lymph node metastasis. ${ }^{16,17}$ The 5-and 10-year overall survival rates are $54 \%$ and $27 \%$, respectively, with standard treatment. ${ }^{16}$ Patients with stage cT1 micropapillary BCA frequently die from disease, and mortality may be reduced by early cystectomy. In the largest single-institution study of stage cT1 micropapillary carcinoma to date $(n=72)$, Willis et $\mathrm{al}^{18}$ showed that patients receiving early cystectomy had 100\% 5-year diseasespecific survival (DSS) compared with 60\% 5-year DSS in conservatively managed patients $(P=.006)$. Similarly, using data from the National Cancer Data Base, Sui et $\mathrm{al}^{19}$ showed that cystectomy improves survival compared with bladder-conserving surgery in stage cT1 micropapillary BCA, although this finding only approached statistical significance $(P=.057)$. Separate, smaller studies have not corroborated a mortality reduction with early cystectomy, although they support that stage cT1 micropapillary carcinoma has high mortality even on short-term follow-up. ${ }^{20,21}$ Given these data, many advocate early cystectomy in stage cT1 micropapillary cancers, ${ }^{17,18,22}$ although this recommendation is not universal.

NAC often induces cPR in micropapillary BCA, although a direct survival benefit has not been demonstrated. ${ }^{23,24}$ In the largest single-institution study to date $(\mathrm{n}=103)$, Fernandez et $\mathrm{al}^{23}$ showed $\mathrm{cPR}$ in $52 \%$ of micropapillary carcinomas treated with NAC compared with $19 \%$ of micropapillary carcinomas treated with immediate cystectomy $(P=.002)$. The 5 -year DSS rate was $96 \%$ in patients with cPR compared with $45 \%$ in patients without such response $(P<.001)$. However, superior survival was not directly reported in the NAC group. In a study using the National Cancer Data Base, no survival benefit was found in micropapillary BCA. ${ }^{19}$ The combined data provide an unclear answer as to the value of NAC in micropapillary BCA, although the frequency of $\mathrm{cPR}$ to chemotherapy and the improved survival in such patients suggest that it is appropriate for this variant.

Diagnostic reproducibility of micropapillary carcinoma is only moderate among pathologists. ${ }^{25}$ Specifically, although diagnostic reproducibility is high in cases of classic micropapillary carcinoma (seen as small nests with surrounding tissue retraction), diagnostic reproducibility is low in cases composed of "admixed large and small cell nests with retraction." 25 Some consider this pattern as micropapillary, whereas others do not. In the cystectomy setting, high rates of nodal metastasis are seen in both classic micropapillary BCA and invasive carcinoma with "admixed large and small cell nests with retraction," suggesting that both patterns similarly inform on tumor behavior. ${ }^{26}$ However, no study has evaluated invasive carcinoma with "admixed large and small cell nests with retraction" in the transurethral resection setting. Thus, it remains unclear whether such cases should receive early cystectomy, and ongoing work aims to clarify this issue.

\section{Plasmacytoid}

Plasmacytoid morphology describes a pattern of infiltrative, individual tumor cells, morphologically 
similar to lobular breast cancer and gastric signetring cancer. ${ }^{7,27}$ In keeping with this aggressive histology, plasmacytoid BCA is clinically aggressive, with approximately $90 \%$ of cases extending outside the bladder at diagnosis. ${ }^{28}$ The 5 -year survival rate is $<30 \%{ }^{28}$ This variant has a unique propensity for peritoneal spread. ${ }^{29,30}$ Plasmacytoid morphology is likewise associated with worse disease-free and overall survival compared with conventional UC, even when controlling for tumor stage and nodal status. ${ }^{30}$

Approximately $80 \%$ of cT 1 plasmacytoid BCAs are upstaged to $\geq \mathrm{pT} 3$ disease at cystectomy. ${ }^{31}$ Conservative management of cT1 plasmacytoid cancer thus greatly risks undertreatment, and early definitive management is prudent.

NAC appears less effective in plasmacytoid carcinoma than conventional UC. For example, Keck et $\mathrm{al}^{32}$ showed that plasmacytoid carcinoma has worse median overall survival (27.4 months) than both micropapillary carcinoma (64.2 months) and conventional UC (62.6 months) $(P=.013)$ when treated with NAC and cystectomy. In keeping with this, Dayyani et $\mathrm{al}^{29}$ showed that although patients with plasmacytoid cancers may experience a $\mathrm{cPR}$ to NAC, recurrence is common. The NAC group in this study was small, however, comprising only 5 patients. Presently, no well-powered study has specifically addressed survival in patients with plasmacytoid cancer treated with immediate cystectomy versus NAC plus cystectomy.

Diagnostic interobserver variability has not been studied in plasmacytoid carcinoma to the author's knowledge.

\section{Small Cell}

Small cell carcinoma morphology in BCA describes a high-grade neuroendocrine carcinoma, morphologically like small cell lung cancer. Like its pulmonary cognate, small cell BCA is aggressive, frequently presenting with lymph node metastasis and high tumor stage. ${ }^{33}$ Patients with small cell BCA historically had a 5-year survival rate of $<30 \%$. ${ }^{8,9}$ However, this tumor type responds uniquely well to NAC. In the largest study to date ( $\mathrm{n}=95$ patients), Lynch et $\mathrm{al}^{34}$ showed that patients with small cell BCA receiving NAC have markedly improved 5-year DSS compared with patients receiving upfront cystectomy (79\% vs $20 \% ; P<.001)$. NAC is thus the standard of care for this variant, as reflected in the NCCN Clinical Practice Guidelines in Oncology (NCCN
Guidelines) for BCA. ${ }^{35}$ Notably, the guidelines state that patients with small cell BCA (including those with a component of conventional UC) should receive neoadjuvant cisplatin and etoposide, agents used for primary therapy in small cell lung cancer, instead of the chemotherapeutic regimens typically used for conventional UC. ${ }^{35,36}$

Stage cT1 small cell BCA is rare, ${ }^{8}$ and cases diagnosed with stage cT1 disease at transurethral resection are often understaged..$^{34}$ Given the high risk of understaging and aggressive biology of this variant, definitive treatment with NAC is the standard of care for small cell BCAs, regardless of stage, including cT1 cases (per NCCN Guidelines). ${ }^{35}$

Small cell BCA has a similar morphology to small cell lung cancer, although interobserver variability is not studied to the author's knowledge. Importantly, conventional UC may at times have a "small cell" pattern. These must be distinguished from true small cell BCA. The immunophenotypes of these tumors differ, and immunohistochemistry can resolve most challenging cases. ${ }^{37}$

\section{Squamous}

Squamous differentiation appears histologically as keratin or intercellular bridges in invasive BCA. ${ }^{7,13}$ Squamous differentiation may be incomplete, termed UC with squamous differentiation (UC-SD). This is common, seen in up to $40 \%$ of conventional UCs. ${ }^{?}$ Squamous differentiation may also involve the entire tumor, termed pure squamous cell carcinoma (SCC) of the bladder. This is classically seen in countries with endemic schistosomiasis, a well-described risk factor. ${ }^{7}$ It is also seen in Western countries, ${ }^{38}$ particularly in patients with chronic catheterization. $.^{39} \mathrm{UC}-\mathrm{SD}$ and pure SCC of the bladder are typically considered separate entities. ${ }^{\text {? }}$

UC-SD tends to be high stage at diagnosis, although survival does not differ between UC-SD and conventional UC when controlling for tumor stage and patient age. ${ }^{40}$ To the author's knowledge, no study has shown that stage cT1 UC-SD responds worse to conservative management than conventional UC. Therefore, treatment decisions in cT1 disease are challenging. On one hand, UC-SD is often high-stage, suggesting that patients with cT1 disease are at great risk of understaging, and should thus be treated aggressively. On the other hand, squamous differentiation is present in a large frac- 
Warrick

tion of invasive BCAs, and no study has shown that patients with UC-SD will do worse than those with conventional UC following conservative management. A reasonable approach is to consider patients with stage CT1 UC-SD as candidates for conservative management, provided staging is thorough and they have no other risk factors for progression and death from disease. ${ }^{41}$

Pure SCC of the bladder typically presents at high stage, and is thus treated aggressively. ${ }^{42}$ However, management of cT1 pure SCC is less clear, largely because it is rare..$^{38,43}$ Using SEER data to address this, Scosyrev et $\mathrm{al}^{44}$ showed that patients with stage cT1 pure SCC had higher 2-year mortality than patients with cT1 conventional UC, if managed conservatively $(P<.001)$. In contrast, mortality did not differ between pure SCC and conventional UC if patients with stage cT1 disease were treated with cystectomy. Based on this study and others showing high mortality and risk of recurrence in pure SCC, many clinicians advocate early cystectomy in stage cT1 pure SCC.

Data on the impact of squamous differentiation on chemotherapy response have been contradictory. Older studies of SCC of the bladder showed poor response to chemotherapy compared with pure UC. ${ }^{45}$ For a time, BCAs with squamous differentiation were thus considered largely chemoresistant. However, more recent studies have shown that patients with BCA with squamous differentiation may benefit from chemotherapy. For example, Scosyrev et $\mathrm{al}^{46}$ showed neoadjuvant MVAC reduces mortality in "mixed tumors," defined as BCA with either squamous and/or glandular differentiation, in a retrospective study. Others have reproduced this finding. ${ }^{47}$ Current evidence thus indicates NAC is appropriate in patients with UC-SD. No evidence shows that NAC improves survival in pure SCC of the bladder, as reflected in current NCCN Guidelines. ${ }^{35}$

The distinction between UC-SD and pure SCC may be difficult. Partial sampling of UC-SD at transurethral resection may give the false impression of pure SCC if only the squamous component is sampled. Additionally, some cases are difficult to confidently classify as pure SCC or UC-SD based on histologic review, particularly poorly differentiated carcinomas with extensive squamous differentiation. Given the treatment differences between pure SCC and UC-SD, diagnosis and treatment are best decided by a multidisciplinary team, in which histology and clinical data are synthesized into a cogent diagnosis and management plan. For example, a patient found to have muscle-invasive BCA with complete squamous differentiation on transurethral resection should not necessarily be diagnosed with pure SCC, given the potential for undersampling, especially in the absence of risk factors for pure SCC. Such a patient could be a candidate for NAC if the full clinicopathologic picture pointed more toward UC-SD.

\section{Glandular}

Glandular differentiation is morphologically defined as areas of carcinoma with the appearance of glandforming adenocarcinoma. ${ }^{7}$ Similar to squamous differentiation, BCAs with complete glandular differentiation are considered pure adenocarcinoma, whereas those with admixed UC and gland-forming carcinoma are considered UC with glandular differentiation. ${ }^{7}$ Pure adenocarcinoma of the bladder is rare, and enriched in patients with schistosomiasis and bladder extrophy. ${ }^{\text {? }}$

Although glandular differentiation in UC is associated with high-stage disease, it is not predictive of survival when accounting for tumor stage and patient age. ${ }^{40}$ To the author's knowledge, glandular differentiation in cT1 BCA has not been shown to predict poor outcomes with conservative management. As with squamous differentiation, glandular differentiation alone does not appear to be an indication for early cystectomy in patients with cT1 BCA, provided staging is thorough and there are no other risk factors. ${ }^{41}$ That stated, this situation is uncommon.

Pure adenocarcinoma of the bladder typically presents at high stage, and is associated with high rates of local recurrence and metastasis. ${ }^{48}$ Treatment is thus radical cystectomy in most cases. Management is not well-studied in cT1 bladder adenocarcinoma because it is rare. ${ }^{48,49}$ However, given the association between adenocarcinoma and aggressive disease, many advocate definitive cystectomy in earlystage nonurachal bladder adenocarcinoma. ${ }^{41}$

As stated earlier, NAC reduces mortality in BCA with squamous or glandular differentiation, indicating that glandular differentiation is not a contraindication to NAC. ${ }^{46,47}$ No strong evidence shows that NAC improves survival in pure adenocarcinoma of the bladder, as reflected in current NCCN Guidelines..$^{35}$

As with squamous differentiation in BCA, distinguishing pure adenocarcinoma from UC with 
extensive glandular differentiation may be difficult in some cases because of sampling issues and when the histologic patterns are difficult to interpret. Diagnosis and treatment are best decided by a multidisciplinary team, in which histology and clinical information are used together to determine the best diagnosis and treatment plan.

\section{Nested}

Nested carcinoma tends to be high stage at presentation, but is not an independent predictor of survival when accounting for tumor stage. ${ }^{50}$ The current value in recognizing nested carcinoma lies in preventing misdiagnosis. Nested carcinomas are histologically seen as invasive cancer nests with minimal cytologic atypia ${ }^{7,51}$ and therefore may appear deceptively similar to benign Vonn Brunn nests, particularly on superficial biopsies. ${ }^{52}$ Likewise, the invasive "large nested" variant of UC may appear deceptively similar to noninvasive low-grade papillary UC. ${ }^{53}$ No study to the author's knowledge has addressed outcomes in conservatively managed stage cT1 nested cancers, nor has a study investigated NAC response in this variant.

\section{Lymphoepithelioma-Like}

The lymphoepithelioma-like variant describes BCA with a histology similar to nonkeratinizing nasopharyngeal carcinoma, specifically undifferentiated carcinoma with large pleomorphic nuclei, prominent nucleoli, and indistinct cell borders, associated with a prominent lymphocytic proliferation. ${ }^{7}$ Lymphoepithelioma-like BCAs are typically high-stage, like other histologic variants. ${ }^{54-58}$ Despite histologic similarity, lymphoepithelioma-like BCA lacks association with Epstein-Barr virus infection, a known association in nasopharyngeal carcinoma..$^{57-59}$
Several small studies ( $<10$ cases) have suggested that patients with high-stage BCA with pure or predominant lymphoepithelioma-like histology may be adequately treated with chemotherapy and transurethral resection of the tumor, foregoing cystectomy. ${ }^{54-56}$ Although these studies are compelling, a larger study $(n=28)$ showed that patients with lymphoepitheliomalike bladder cancer treated with cystectomy had similar survival to those with muscle-invasive conventional UC, and that those treated with chemotherapy and transurethral resection may experience recurrence. ${ }^{57}$ Although it is possible that a subset of patients with pure lymphoepithelioma-like BCA may be safely treated with chemotherapy and transurethral resection, the data are insufficient to justify this as a treatment recommendation. Diagnostic reproducibility among pathologists is also not studied in the lymphoepithelioma-like variant, and will be important if this variant is ultimately used to guide therapy.

\section{Conclusions}

Histologic variants of $\mathrm{BCA}$ are emerging as valuable biomarkers for clinical decision-making. Although several issues remain unresolved, pathologists and treating physicians will benefit from understanding these variants and their specific prognostic and therapeutic indications.

\section{Acknowledgments}

The author would like to thank Drs. David DeGraff, Jay Raman, Monika Joshi, and Peter Clark for their guidance in preparing this manuscript.

\section{References}

1. Brausi $\mathrm{M}$, Witjes JA, Lamm D, et al. A review of current guidelines and best practice recommendations for the management of nonmuscle invasive bladder cancer by the International Bladder Cancer Group. J Urol 2011;186:2158-2167.

2. Leblanc B, Duclos AJ, Benard F, et al. Long-term followup of initial Ta grade 1 transitional cell carcinoma of the bladder. J Urol 1999;162:1946-1950.

3. Stein JP, Penson DF. Invasive T1 bladder cancer: indications and rationale for radical cystectomy. BJU Int 2008;102:270-275.

4. Chou R, Selph SS, Buckley DI, et al. Treatment of muscle-invasive bladder cancer: a systematic review. Cancer 2016;122:842-851.

5. Petrelli F, Coinu A, Cabiddu M, et al. Correlation of pathologic complete response with survival after neoadjuvant chemotherapy in bladder cancer treated with cystectomy: a meta-analysis. Eur Urol 2014;65:350-357.

6. Moschini M, Dell'Oglio P, Luciano R, et al. Incidence and effect of variant histology on oncological outcomes in patients with bladder cancer treated with radical cystectomy. Urol Oncol 2017;35:335-341.

7. Moch H, Humphrey PA, Ulbright TM, Reuter VE. WHO Classification of Tumours of the Urinary System and Male Genital Organs. Lyon, France: IARC; 2016.

8. Cheng L, Pan CX, Yang XJ, et al. Small cell carcinoma of the urinary bladder: a clinicopathologic analysis of 64 patients. Cancer 2004;101:957-962.

9. Choong NW, Quevedo JF, Kaur JS. Small cell carcinoma of the urinary bladder. The Mayo Clinic experience. Cancer 2005;103:1172-1178.

10. Comperat E, Roupret M, Yaxley J, et al. Micropapillary urothelial carcinoma of the urinary bladder: a clinicopathological analysis of 72 cases. Pathology 2010;42:650-654.

11. Nigwekar $P$, Tamboli $P, A$ min MB, et al. Plasmacytoid urothelial carcinoma: detailed analysis of morphology with clinicopathologic correlation in 17 cases. Am J Surg Pathol 2009;33:417-424. 
Warrick

12. Alvarado-Cabrero I, Sierra-Santiesteban FI, Mantilla-Morales A, Hernandez-Hernandez DM. Micropapillary carcinoma of the urothelial tract. A clinicopathologic study of 38 cases. Ann Diagn Pathol 2005;9:1-5.

13. Amin MB. Histological variants of urothelial carcinoma: diagnostic, therapeutic and prognostic implications. Mod Pathol 2009;22(Suppl 2):S96-118.

14. Amin MB, Eble JN. Urological Pathology, 1st ed. Philadelphia, PA: Wolters Kluwer Health/Lippincott Williams \& Wilkins; 2014.

15. Epstein JI, Amin MB, Reuter VE, Epstein JI. Biopsy Interpretation of the Bladder, 2nd ed. Philadelphia, PA: Wolters Kluwer/Lippincott Williams \& Wilkins Health; 2010.

16. Kamat AM, Dinney CP, Gee JR, et al. Micropapillary bladder cancer: a review of the University of Texas M. D. Anderson Cancer Center experience with 100 consecutive patients. Cancer 2007;110:62-67.

17. Kamat AM, Gee JR, Dinney CP, et al. The case for early cystectomy in the treatment of nonmuscle invasive micropapillary bladder carcinoma. J Urol 2006;175(3 Pt 1):881-885.

18. Willis DL, Fernandez MI, Dickstein RJ, et al. Clinical outcomes of cT1 micropapillary bladder cancer. J Urol 2015;193:1129-1134.

19. Sui W, Matulay JT, James MB, et al. Micropapillary bladder cancer: insights from the National Cancer Database. Bladder Cancer 2016;2:415-423.

20. Gaya JM, Palou J, Algaba F, et al. The case for conservative management in the treatment of patients with non-muscle-invasive micropapillary bladder carcinoma without carcinoma in situ. Can J Urol 2010;17:5370-5376.

21. Spaliviero $M$, Dalbagni $G$, Bochner $B H$, et al. Clinical outcome of patients with T1 micropapillary urothelial carcinoma of the bladder. J Urol 2014;192:702-707.

22. Babjuk M, Burger M, Zigeuner R, et al. EAU guidelines on non-muscleinvasive urothelial carcinoma of the bladder: update 2013. Eur Urol 2013;64:639-653.

23. Fernandez MI, Williams SB, Willis DL, et al. Clinical risk stratification in patients with surgically resectable micropapillary bladder cancer. BJU Int 2017;119:684-691.

24. Meeks JJ, Taylor JM, Matsushita K, et al. Pathological response to neoadjuvant chemotherapy for muscle-invasive micropapillary bladder cancer. BJU Int 2013;111:E325-330.

25. Sangoi $A R$, Beck $A H, A m i n ~ M B$, et al. Interobserver reproducibility in the diagnosis of invasive micropapillary carcinoma of the urinary tract among urologic pathologists. Am J Surg Pathol 2010;34:1367-1376.

26. Shah TS, Kaag M, Raman JD, et al. Clinical significance of prominent retraction clefts in invasive urothelial carcinoma. Hum Pathol 2017;61:9096.

27. Mai KT, Park PC, Yazdi HM, et al. Plasmacytoid urothelial carcinoma of the urinary bladder report of seven new cases. Eur Urol 2006;50:1111-1114.

28. Keck B, Stoehr R, Wach S, et al. The plasmacytoid carcinoma of the bladder-rare variant of aggressive urothelial carcinoma. Int J Cancer 2011;129:346-354.

29. Dayyani F, Czerniak BA, Sircar K, et al. Plasmacytoid urothelial carcinoma, a chemosensitive cancer with poor prognosis, and peritoneal carcinomatosis. J Urol 2013;189:1656-1661.

30. Kaimakliotis HZ, Monn MF, Cheng L, et al. Plasmacytoid bladder cancer: variant histology with aggressive behavior and a new mode of invasion along fascial planes. Urology 2014;83:1112-1116.

31. Kaimakliotis HZ, Monn MF, Cary KC, et al. Plasmacytoid variant urothelial bladder cancer: is it time to update the treatment paradigm? Urol Oncol 2014;32:833-838.

32. Keck B, Wach S, Stoehr R, et al. Plasmacytoid variant of bladder cancer defines patients with poor prognosis if treated with cystectomy and adjuvant cisplatin-based chemotherapy. BMC Cancer 2013;13:71.

33. Trias I, Algaba F, Condom E, et al. Small cell carcinoma of the urinary bladder. Presentation of 23 cases and review of 134 published cases. Eur Urol 2001;39:85-90.

34. Lynch SP, Shen Y, Kamat A, et al. Neoadjuvant chemotherapy in small cell urothelial cancer improves pathologic downstaging and long-term outcomes: results from a retrospective study at the MD Anderson Cancer Center. Eur Urol 2013;64:307-313.

35. Spiess PE, Agarwal N, Bangs R, et al. NCCN Clinical Practice Guidelines in Oncology: Bladder Cancer, Version 5.2017. Accessed July 8, 2017. To view the most recent version of these guidelines, visit NCCN.org.

36. Kalemkerian GP, Loo BW Jr, Akerley W, et al. NCCN Clinical Practice Guidelines in Oncology: Small Cell Lung Cancer, Version 3.2017. Accessed July 8,2017 . To view the most recent version of these guidelines, visit NCCN.org.
37. ThompsonS, Cioffi-LavinaM,Chapman-FredricksJ, etal. Distinction of high grade neuroendocrine carcinoma/small cell carcinoma from conventional urothelial carcinoma of urinary bladder: an immunohistochemical approach Appl Immunohistochem Mol Morphol 2011;19:395-399.

38. Lagwinski N, Thomas A, Stephenson AJ, et al. Squamous cell carcinoma of the bladder: a clinicopathologic analysis of 45 cases. Am J Surg Pathol 2007;31:1777-1787.

39. Broecker BH, Klein FA, Hackler RH. Cancer of the bladder in spinal cord injury patients. J Urol 1981;125:196-197.

40. Mitra AP, Bartsch CC, Bartsch G Jr, et al. Does presence of squamous and glandular differentiation in urothelial carcinoma of the bladder at cystectomy portend poor prognosis? An intensive case-control analysis. Urol Oncol 2014;32:117-127.

41. Porten SP, Willis D, Kamat AM. Variant histology: role in management and prognosis of nonmuscle invasive bladder cancer. Curr Opin Urol 2014;24:517-523.

42. Kassouf W, Spiess PE, Siefker-Radtke A, et al. Outcome and patterns of recurrence of nonbilharzial pure squamous cell carcinoma of the bladder: a contemporary review of The University of Texas M D Anderson Cancer Center experience. Cancer 2007;110:764-769.

43. Abdollah F, Sun $M$, Jeldres C, et al. Survival after radical cystectomy of nonbilharzial squamous cell carcinoma vs urothelial carcinoma: a competingrisks analysis. BJU Int 2012;109:564-569.

44. Scosyrev E, Yao J, Messing E. Urothelial carcinoma versus squamous cell carcinoma of bladder: is survival different with stage adjustment? Urology 2009;73:822-827.

45. Clark PE. Urothelial carcinoma with squamous differentiation: response to chemotherapy and radiation. Urol Oncol 2015;33:434-436.

46. Scosyrev E, Ely BW, Messing EM, et al. Do mixed histological features affect survival benefit from neoadjuvant platinum-based combination chemotherapy in patients with locally advanced bladder cancer? A secondary analysis of Southwest Oncology Group-Directed Intergroup Study (S8710). BJU Int 2011;108:693-699.

47. Zargar-Shoshtari K, Sverrisson EF, Sharma P, et al. Clinical outcomes after neoadjuvant chemotherapy and radical cystectomy in the presence of urothelial carcinoma of the bladder with squamous or glandular differentiation. Clin Genitourin Cancer 2016;14:82-88.

48. Grignon DJ, Ro JY, Ayala AG, et al. Primary adenocarcinoma of the urinary bladder. A clinicopathologic analysis of 72 cases. Cancer 1991;67:21652172.

49. Zaghloul MS, Nouh A, Nazmy M, et al. Long-term results of primary adenocarcinoma of the urinary bladder: a report on 192 patients. Urol Oncol 2006;24:13-20.

50. Beltran AL, Cheng L, Montironi R, et al. Clinicopathological characteristics and outcome of nested carcinoma of the urinary bladder. Virchows Arch 2014;465:199-205.

51. Drew PA, Furman J, Civantos F, Murphy WM. The nested variant of transitional cell carcinoma: an aggressive neoplasm with innocuous histology. Mod Pathol 1996;9:989-994.

52. Volmar KE, Chan TY, De Marzo AM, Epstein JI. Florid von Brunn nests mimicking urothelial carcinoma: a morphologic and immunohistochemical comparison to the nested variant of urothelial carcinoma. Am J Surg Pathol 2003;27:1243-1252.

53. Cox R, Epstein JI. Large nested variant of urothelial carcinoma: 23 cases mimicking von Brunn nests and inverted growth pattern of noninvasive papillary urothelial carcinoma. Am J Surg Pathol 2011;35:1337-1342.

54. Amin MB, Ro JY, Lee KM, et al. Lymphoepithelioma-like carcinoma of the urinary bladder. Am J Surg Pathol 1994;18:466-473.

55. Holmang S, Borghede G, Johansson SL. Bladder carcinoma with lymphoepithelioma-like differentiation: a report of 9 cases. J Urol 1998;159:779-782.

56. Lopez-Beltran A, Luque RJ, Vicioso L, et al. Lymphoepithelioma-like carcinoma of the urinary bladder: a clinicopathologic study of 13 cases. Virchows Arch 2001;438:552-557.

57. Tamas EF, Nielsen ME, Schoenberg MP, Epstein JI. Lymphoepithelioma-like carcinoma of the urinary tract: a clinicopathological study of 30 pure and mixed cases. Mod Pathol 2007;20:828-834.

58. Williamson SR, Zhang S, Lopez-Beltran A, et al. Lymphoepithelioma-like carcinoma of the urinary bladder: clinicopathologic, immunohistochemical, and molecular features. Am J Surg Pathol 2011;35:474-483.

59. Gulley ML, Amin MB, Nicholls JM, et al. Epstein-Barr virus is detected in undifferentiated nasopharyngeal carcinoma but not in lymphoepitheliomalike carcinoma of the urinary bladder. Hum Pathol 1995;26:1207-1214. 